\title{
Power Spectra of Nonlinearly Coupled Waves
}

\author{
J. Sherman and J. McLaughlin \\ Department of Physics, Clarkson College of Technology, Potsdam, New York 13676, USA
}

\begin{abstract}
Swinney et al. (1977) have found that turbulence in rotational Couette flow results from a small number of instabilities. They have raised the question of whether these instabilities conform with the ideas of Ruelle and Takens (1971). We show that a simple model of the Couette flow yields power spectra similar to those seen in the experiments. The model is consistent with the Ruelle and Takens picture.
\end{abstract}

\section{Introduction}

Recent experiments on circular Couette flow by Gollub and Swinney [1] and Swinney, Fenstermacher, and Gollub (SFG) [2] have revealed a series of transitions in the power spectrum of the radial component of velocity at a point midway between the two cylinders. At low Reynolds numbers, these investigators find spectra consisting of spikes. The spectra correspond to periodic or quasiperiodic motions of the fluid. At a well defined Reynolds number, the spikes disappear leaving a series of broad humps in the power spectrum. (Only one hump was visible in the earlier experiment.) It has been asked whether these broad power spectra might be produced by a "strange-attractor" $[3,4]$ solution of the governing equation. We have constructed a simple model of nonlinearly coupled waves which has stochastic solutions when three or more waves have sufficiently positive growth rates. The power spectra of these systems is qualitatively similar to those observed by SFG.

\section{The Model}

A realistic theoretical study of the Couette flow transitions observed by SFG would require solution of the three-dimensional Navier Stokes equation. However, it is of some interest to investigate whether nonlinear coupling between a small number of waves could yield broad power spectra similar to those observed by SFG. Photographs of the flows studied by SFG reveal phenomena similar to those observed by Coles [5]. At low rotations speeds, the flow is azimuthal. As the Reynolds number is increased, Taylor cells appear in the flow. The next instability 


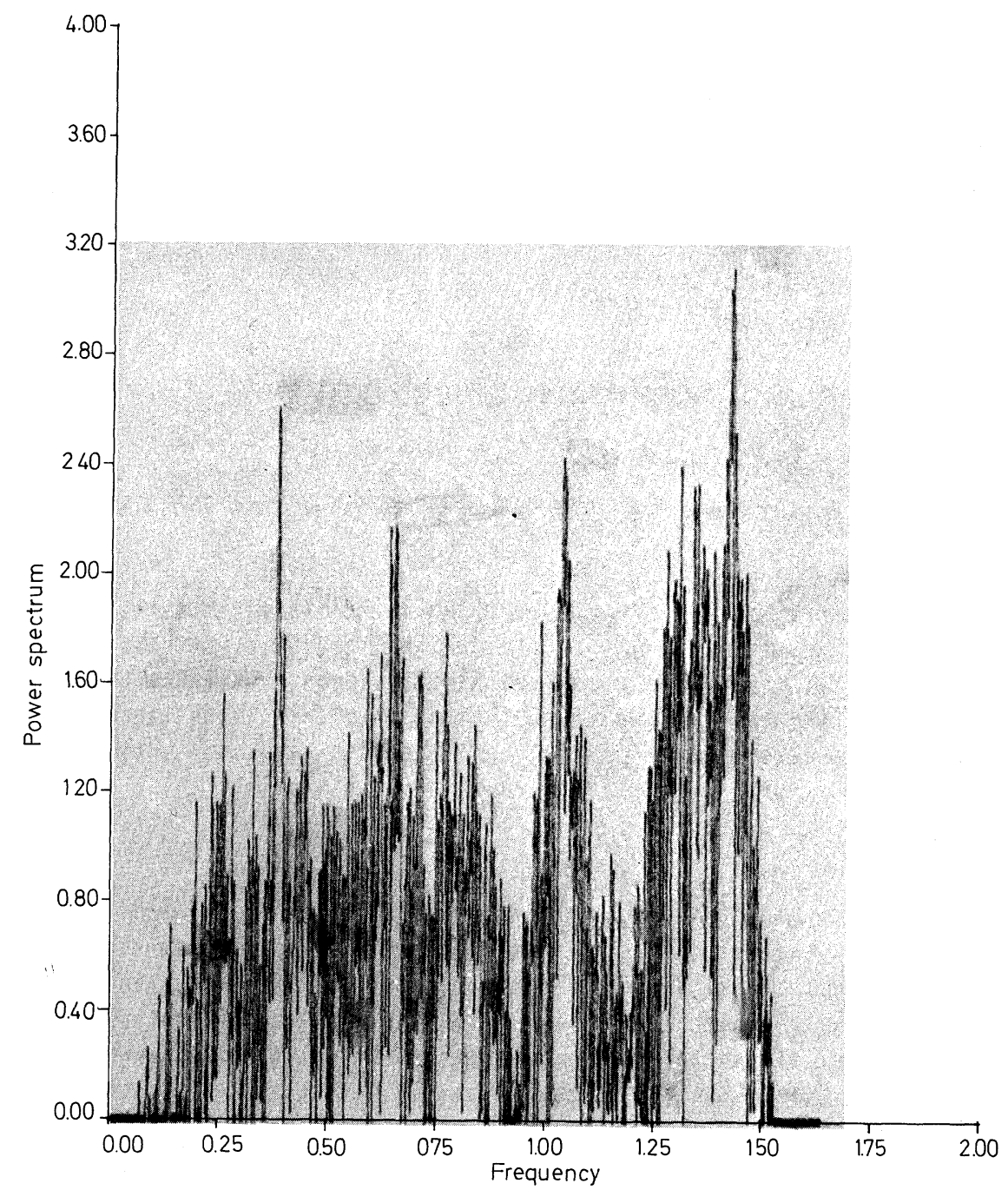

Fig. 1. Power spectrum for $\omega_{2}^{i}=0.4, \omega_{3}^{i}=0.3, \omega_{4}^{i}=0.5$

leads to "wavy" Taylor cells. The waves propagate in the azimuthal direction. The waves show up as a spike (and its harmonics) in the power spectrum.

The humps in the broad spectrum are centered on fairly well defined frequencies. This suggests that a finite number of coupled waves may be broadening their spectra through the type of motion discussed by Ruelle and Takens [3]. To investigate this possiblity, let us construct a two-dimensional (radial and azimuthal variations) model of the Coles waves. The fluid motion can be calculated from a stream function which obeys the nonlinear Orr-Sommerfeld equation [6].

$$
\begin{aligned}
& \psi=\psi_{L}^{(r)}+\psi_{F}(r, \phi, t), \\
& \frac{\partial}{\partial t}\left(\mathscr{L}_{1}\left(\psi_{F}\right)\right)=\mathscr{L}_{2}\left(\psi_{F}\right)+\mathscr{L}_{3}\left(\psi_{F}^{2}\right) .
\end{aligned}
$$




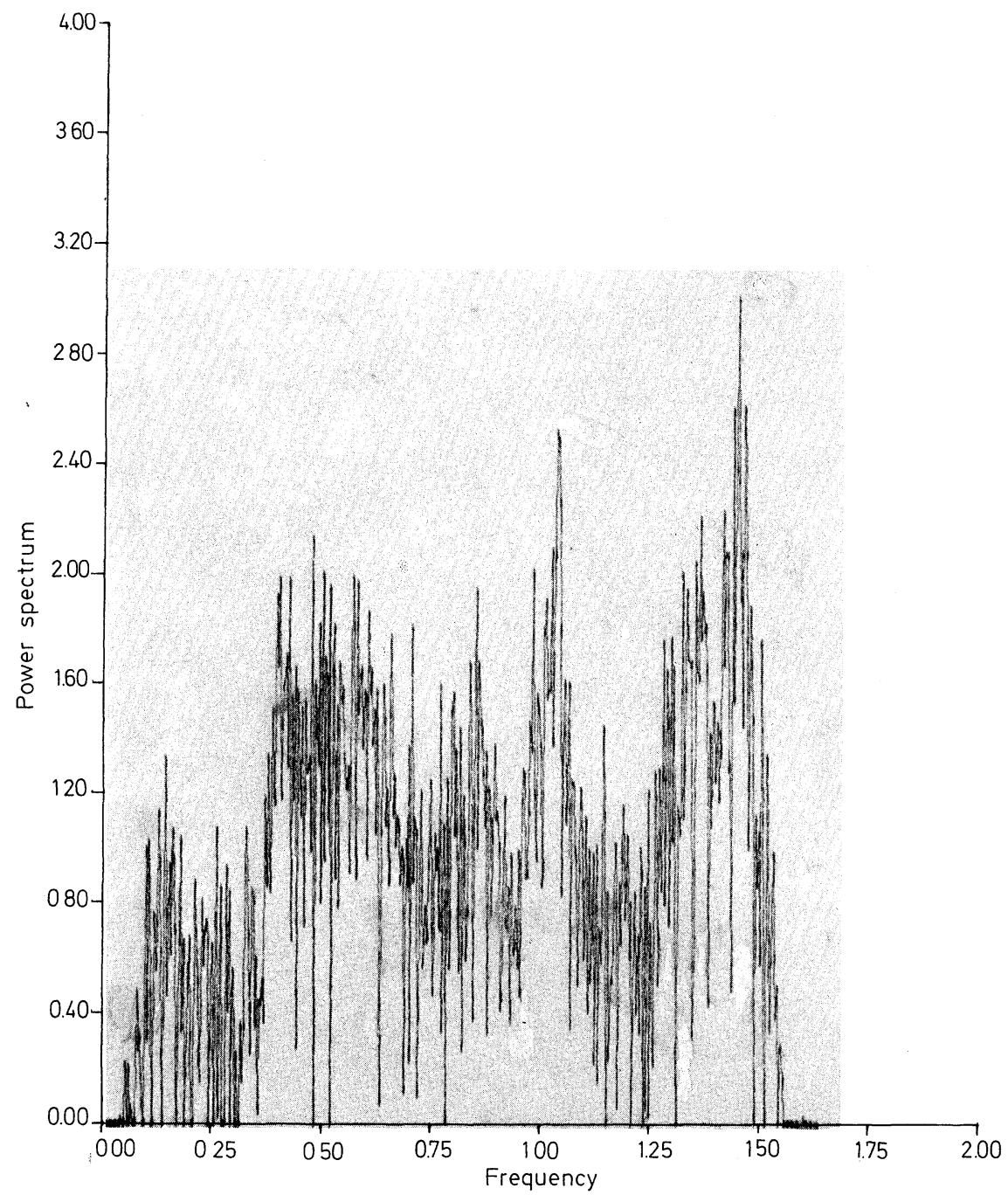

Fig. 2. Power spectrum for $\omega_{2}^{i}=0.5, \omega_{3}^{i}=0.4, \omega_{4}^{i}=0.6$

In Equations (1) and (2), $\psi_{L}$ is the stream function for a laminar flow between the circles, $\psi$ is the stream function for the time dependent flow, and $\mathscr{L}_{i}$ are linear operators. Schensted [6] has shown that the eigenfunctions of the linearized OrrSommerfeld equation for a given boundary value problem form a complete set. Thus, one can make the following decomposition:

$$
\begin{aligned}
& \psi_{F}^{(r, \phi)}=\sum_{m n} A_{m n}(t) F_{m n}(r) e^{i n \phi} \equiv \sum_{m n} A_{m n}(t) \psi_{m n}, \\
& \mathscr{L}_{2}\left(\psi_{m n}\right)=-i \omega_{m n} \mathscr{L}_{1}\left(\psi_{m n}\right) .
\end{aligned}
$$

A set of ODE's for the amplitudes $A_{m, n}$ can be projected out by taking inner products of Equation (2) with the eigenfunctions of the adjoint linearized Orr- 


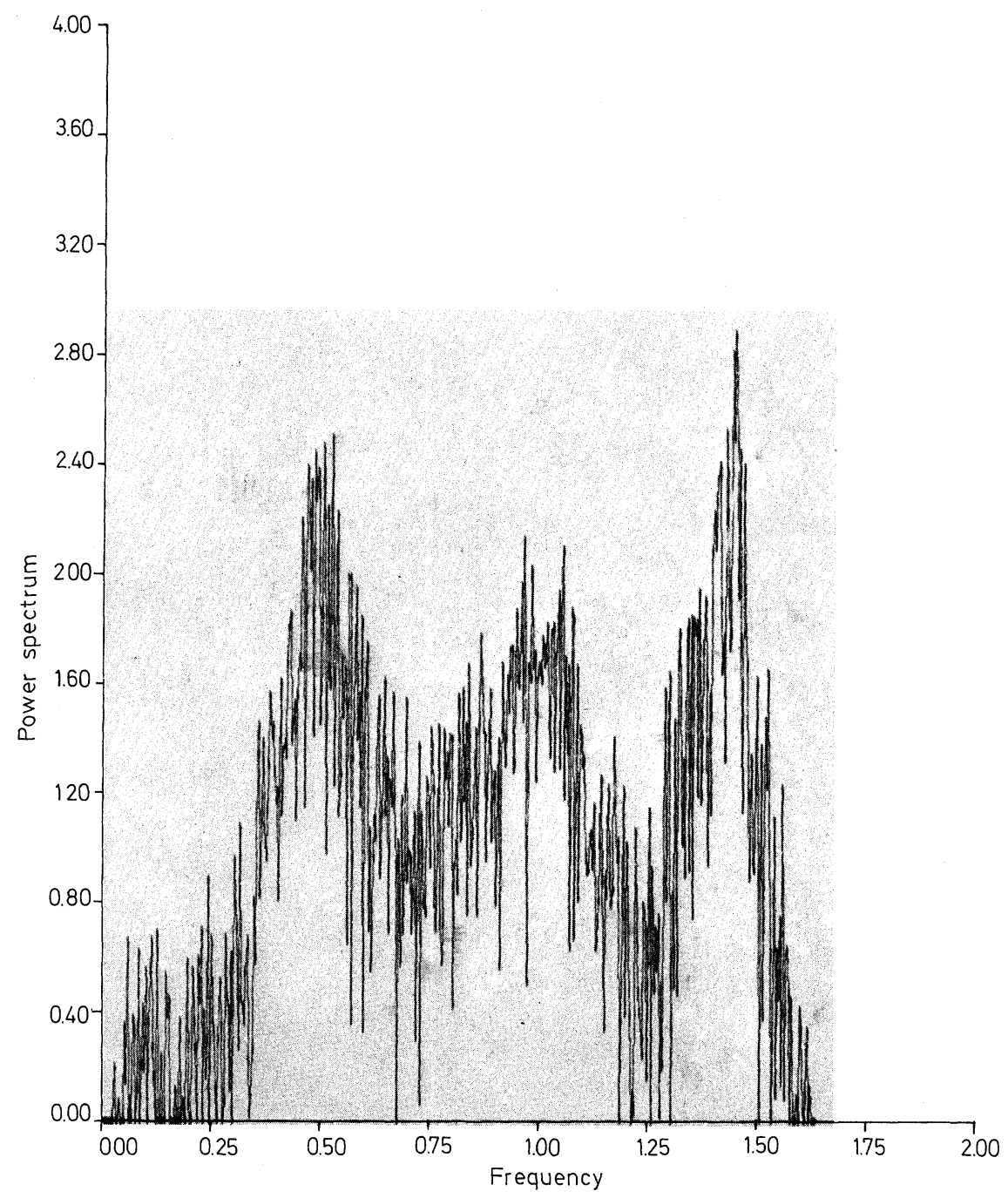

Fig. 3. Power spectrum for $\omega_{2}^{i}=0.6, \omega_{3}^{i}=0.5, \omega_{4}^{i}=0.7$

Sommerfeld equation [6], $\psi_{m n}^{+}(r, \phi)$.

$$
\begin{aligned}
& \frac{d A_{m n}}{d t}=\sum_{p, q, r} \gamma(m, n, p, q, r) A_{p q} A_{r, n-q}-i \omega_{m n} A_{m n}, \\
& \gamma(m, n, p, q, r) \equiv \int \psi_{m n}^{\dagger} \mathscr{L}_{3}\left(\psi_{p q} \psi_{r, n-q}\right) .
\end{aligned}
$$

Let us now introduce some simplifications. First, let us eliminate all branches of the dispersion relation except the lowest $(m=1)$. Next, instead of trying to calculate the $\gamma$ 's, let us set them equal to $i$, and investigate the behaviour of the following systems

$$
\frac{d A_{n}}{d t}=i \sum_{q} A_{q} A_{n-q}-i \omega_{n} A_{n} .
$$

This svstem will be truncated to include onlv $0 \leq n \leq 4$. 


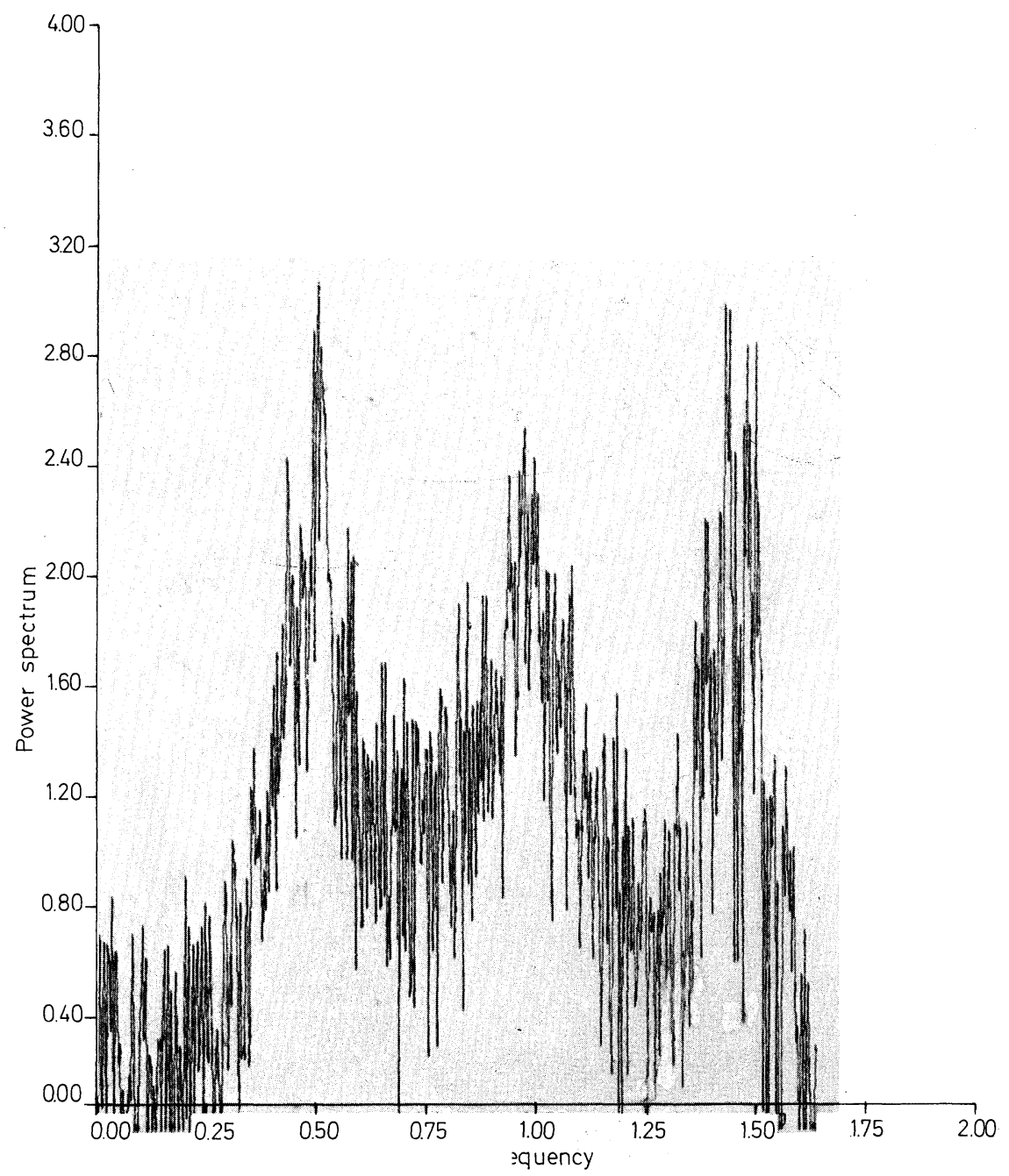

Fig. 4. Power spectrum for $\omega_{2}^{t}=0.7, \omega_{3}^{t}=0.6, \omega_{4}^{t}=0.8$

\section{Choice of Parameters}

The frequencies in Equation (7) were chosen to facilitate numerical calculation, and to produce humps centered on frequencies which have roughly the same ratios as those measured in the SFG experiments

$$
\begin{aligned}
& \omega_{n}=\omega_{n}^{r}+i \omega_{n}^{i}, \\
& \omega_{n}^{r}=0.6 n+0.12 n^{2} .
\end{aligned}
$$

It was found that choosing one or two of the growth rates to be positive while the others were negative always resulted in periodic or quasiperiodic power spectra. However, by choosing three of the growth rates to be sufficiently positive, we were able to obtain broad power spectra similar to those measured by SFG. In all of these runs, we took $\omega_{0}^{i}=-1$ and $\omega_{1}^{i}=0$. Thus, there were only three "active" modes. 


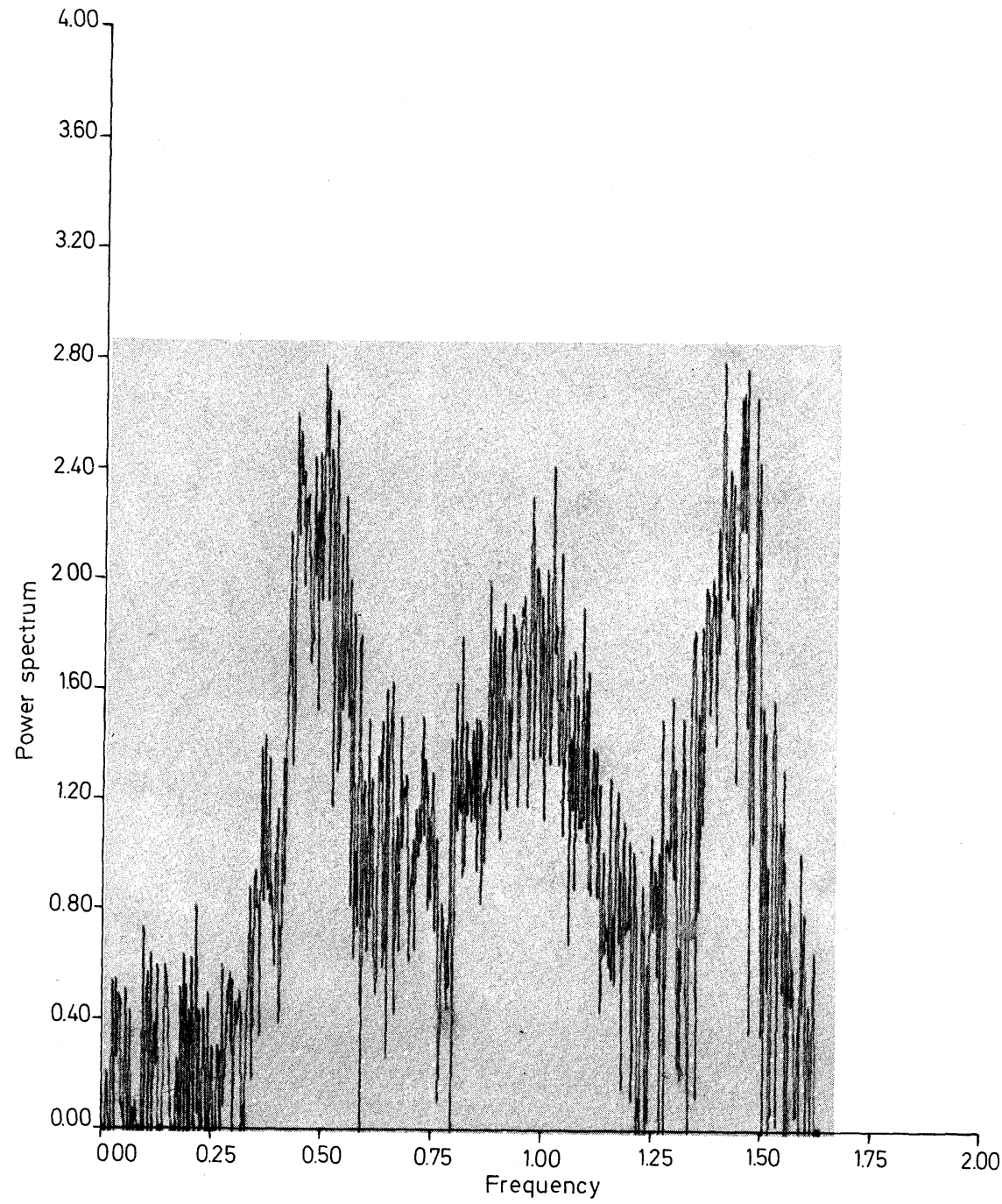

Fig. 5. Power spectrum for $\omega_{2}^{i}=0.8, \omega_{3}^{i}=0.7, \omega_{4}^{i}=0.9$

\section{Numerical Results}

In order to emulate the effect of increasing the Reynolds number in our system, the growth rates for waves 2,3 , and 4 were chosen as follows

$$
\begin{aligned}
& \omega_{2}^{i}=0.4+l(0.1), \\
& \omega_{3}^{i}=0.3+l(0.1), \\
& \omega_{4}^{i}=0.5+l(0.1) .
\end{aligned}
$$

For each choice of $l$, the equations were integrated using a fourth order predictorcorrector scheme. Three different time steps $(0.05,0.025$, and 0.0125$)$ and two time record lengths (600 and 1200) were used for each value of $l$. The power spectra obtained were insensitive to time step and record length. The logarithm 


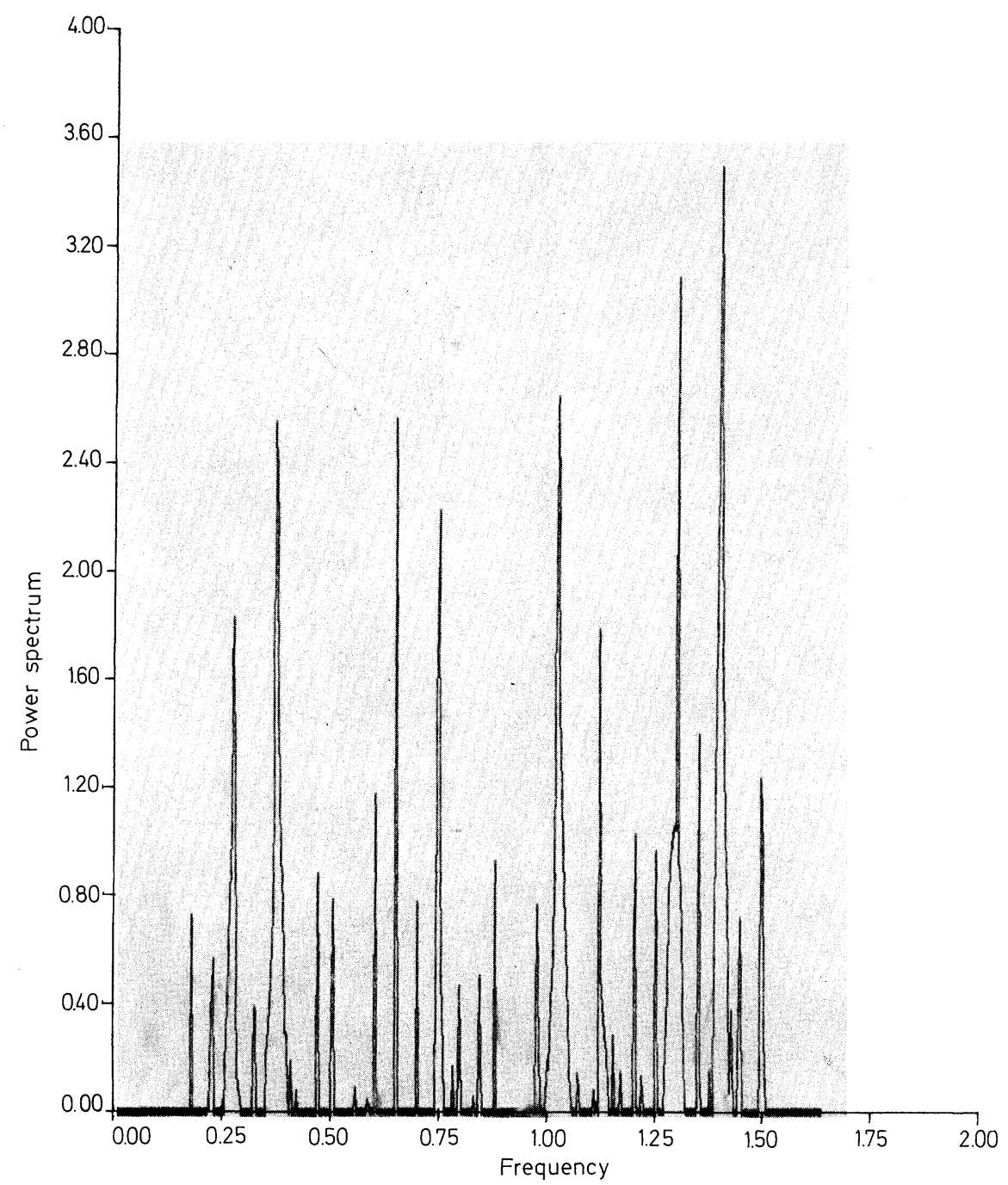

Fig. 6. Power spectrum for $\omega_{2}^{i}=0.3, \omega_{3}^{i}=0.2, \omega_{4}^{i}=0.4$

of the power spectrum for $0 \leqq l \leqq 4$ is graphed as a function of frequency in Figures $1-5$. The frequency axes were scaled by a factor (1/3.2) in order to facilitate comparison with the SFG results.

The power spectrum, $P(\omega)$, of the system was defined as follows:

$$
P(\omega) \equiv \lim _{T \rightarrow \infty} \frac{2 \pi}{T} \sum_{n=0}^{4}\left|\tilde{A}_{n}(\omega, T)\right|^{2},
$$

$$
\tilde{A}_{n}(\omega, T) \equiv \int_{-T / 2}^{T / 2} e^{i \omega t} A_{n}(t) \frac{d t}{2 \pi} .
$$

It will be noticed that the humps in the power spectra tend to broaden as the growth rates increase. The center frequencies change very little. These random solutions are stable in the sense that they exist over wide parameter ranges. The 


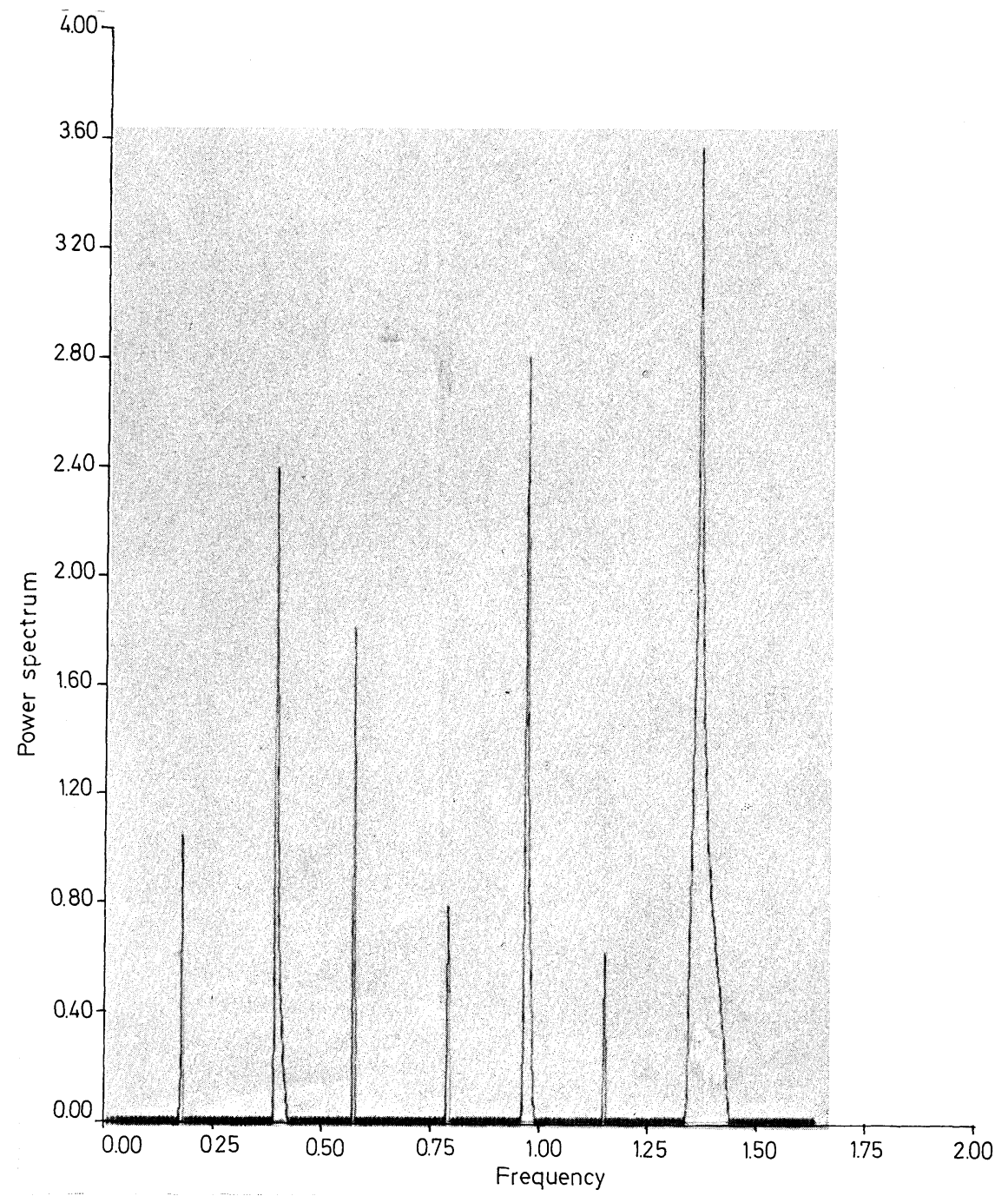

Fig. 7. Power spectrum for $\omega_{2}^{i}=0, \omega_{3}^{i}=0.25, \omega_{4}^{i}=0.4$

relative locations of the humps can be adjusted by varying the coefficient of the quadratic term in Equation (9).

It is of interest that the lower frequencies tend to become more energetic as the growth rates increase. This trend was also noted in the SFG experiments.

We also made runs with smaller growth rates. In Figure 6 we show the results for $l=-1$ in Equations (10-12). It will be seen that a series of spikes is obtained despite the fact that three distinct frequencies are distinguishable. This result may support the theory of Kidachi [7] who maintains that strange attractor behavior occurs only after the waves have achieved a certain threshold amplitude.

Power spectra similar to that in Figure 6 were not seen in the SFG experiments. However, by setting $\omega_{2}^{i}=0, \omega_{3}^{i}=0.25$, and $\omega_{4}^{i}=0.4$, we were able to obtain a quasiperiodic spectrum with spikes in roughly the same relative locations as in the 
SFG experiments for preturbulence Reynolds numbers. This power spectrum is shown in Figure 7.

Of course, the above choices of growth rates are purely ad-hoc. However, all calculations were done using the same dispersion relation for the real part of the linear frequencies [Eq. (9)]. The results suggest a possible interpretation of the SFG results. As the Reynolds number is increased, the four-cycle wave appears first. This corresponds to $\omega_{4}^{i}>0$ and $\omega_{n}^{i}<0$ for $n \neq 4$. Then $\omega_{3}^{i}$ becomes positive and leads to a new set of spikes in the power spectrum. Thus, in this regime two different wavenumbers and their sums and differences are present in the flow. This spectrum corresponds to Figure 7 . Finally $\omega_{2}^{i}$ becomes positive and the spectrum begins to broaden. These spectra are shown in Figures 1-5. It should be possible to check this suggestion by studying the equal time spatial correlations of the flow.

\section{Conclusions}

The results reported above demonstrate the possibility of generating power spectra similar to those seen experimentally through the nonlinear coupling of a small number of waves. This may make it worthwhile to study the spatial variation of the SFG flows in the azimuthal direction. If a small number of waves dominate the power spectrum, there should be a strong spatial correlation in the velocity field.

Acknowledgment. We would like to thank R. Fenstermacher, J. Gollub, and H. Swinney for stimulating discussions and helpful suggestions. This research was supported by NSF Grant No. MPS 7501443 and Research Corporation Grant No. 7552.

\section{References}

1. Gollub,J.P., Swinney,H.L.: Phys. Rev. Letters 35, 14, 927 (1975)

2. Swinney,H.L., Fenstermacher,P.R., Gollub,J.P.:Paper presented at Symposium on Turbulent Shear Flows, April 18-20, 1977

3. Ruelle,D., Takens, F.: Commun. math. Phys. 20, 167 (1971)

4. Lorenz,E.N.: J. atmos. Sci. 20, 130 (1963)

5. Coles, D.: J. Fluid Mech. 21, 385 (1965)

6. Schensted,I.: Contributions to the theory of hydrodynamic stability, Ph.D. Thesis, University of Michigan, 1961

7. Kidachi,H.: A comment on a proposition of D. Ruelle and F. Takens on the nature of turbulence. Preprint, Kyoto University

Communicatẹ by J. L. Lebowitz

Received May 3, 1977 
